\title{
Renormalization in charged colloids: non-monotonic behaviour with the surface charge
}

\author{
C Haro-Pérez ${ }^{1}$, M Quesada-Pérez ${ }^{2}$, J Callejas-Fernández ${ }^{1}$, \\ P Schurtenberger $^{3}$ and R Hidalgo-Álvarez ${ }^{1}$ \\ ${ }^{1}$ Grupo de Física de Fluidos y Biocoloides Departamento de Física, Facultad de Ciencias, \\ University of Granada, 18071 Granada, Spain \\ 2 Departmento de Física, Escuela Politécnica Superior de Linares, University of Jaén, \\ 23700 Linares, Spain \\ ${ }^{3}$ Department of Physics, University of Fribourg, CH-1700 Fribourg, Switzerland \\ E-mail: rhidalgo@ugr.es
}

\begin{abstract}
The static structure factor $S(q)$ is measured for a set of deionized latex dispersions with different numbers of ionizable surface groups per particle and similar diameters. For a given volume fraction, the height of the main peak of $S(q)$, which is a direct measure of the spatial ordering of latex particles, does not increase monotonically with the number of ionizable groups. This behaviour cannot be described using the classical renormalization scheme based on the cell model. We analyse our experimental data using a renormalization model based on the jellium approximation, which predicts the weakening of the spatial order for moderate and large particle charges.
\end{abstract}

A long-standing issue in colloid science is the precise understanding of the electrostatic forces in charge-stabilized dispersions. According to the widely known Derjaguin-Landau-VerweyOverbeek (DLVO) theory, the interaction potential $u(r)$ of charged spherical colloidal particles is

$$
u(r)=\frac{(Z e)^{2}}{4 \pi \varepsilon_{0} \varepsilon_{\mathrm{r}}} \frac{\exp (2 \kappa a)}{(1+\kappa a)^{2}} \frac{\exp (-\kappa r)}{r} .
$$

Here, $Z$ is the number of charges per colloidal particle, $e$ is the elementary charge, $\kappa$ is the Debye-Hückel screening parameter, $\varepsilon_{0} \varepsilon_{\mathrm{r}}$ is the dielectric permittivity of the solvent and $a$ is the colloidal particle radius. This expression was derived assuming small surface charges. However, a large number of theoretical works state that equation (1) could be valid if $Z$ and $\kappa$ are considered as renormalized parameters rather than the actual ones (see [1-3] and references cited therein). Some theories have been devised for predicting renormalized parameters in a 
Table 1. Latexes used in this work.

\begin{tabular}{llll}
\hline Latex & $\begin{array}{l}\text { Diameter } \\
(\mathrm{nm})\end{array}$ & $\begin{array}{l}\text { Surface charge density } \\
\left(\mu \mathrm{C} \mathrm{cm}^{-2}\right)\end{array}$ & $\begin{array}{l}\text { Number of ionizable } \\
\text { groups per particle }\end{array}$ \\
\hline L1 & 82 & 0.63 & 425 \\
L2 & 80 & 1.51 & 2100 \\
L3 & 80 & 2.97 & 4210 \\
L4 & 80 & 7.86 & 11140 \\
\hline
\end{tabular}

numerical way. In the middle 1980s Alexander et al proposed the cell model [4], which has been widely used since then. Accordingly, the dimensionless solution $(\Psi)$ to the PoissonBoltzmann (PB) equation is asymptotically approximated at the boundary of the so-called Wigner-Seitz (WS) cell by the solution of the linearized PB equation $\left(\Psi_{0}\right)$, where the size of the WS cell is determined by the particle concentration. At small distances from the surface, $\Psi_{0}$ underestimates the magnitude and the slope of $\Psi$. This underestimation is interpreted in terms of charge renormalization [4].

At the same time, Beresford-Smith and co-workers [5] developed a renormalization scheme based on the so-called jellium approximation. The theory proposed by these authors is also based on the approximation of $\Psi$ by $\Psi_{0}$ but at large distances in this case. Also in contrast with the cell model, the dispersion is supposed to be completely disordered. In other words, this theory assumes that there are no correlations between the particles, i.e. the colloidcolloid radial distribution function is $g(r)=1$. Although this approach was proposed two decades ago, only recently have a few works emphasized its more peculiar and characteristic predictions. Rojas and co-workers find in their experiments that the amplitude of the first peak of the structure factor does not increase monotonically with the volume fraction $(\varphi)$ but shows a minimum [6], which had already been predicted by Beresford-Smith [5]. Another of its predictions is the behaviour of the structure with particle charge; they reported that the first peak of the structure factor should exhibit a maximum with increasing particle charge. Trizac and Levin propose a renormalized jellium model and argue as to why the effective charge obtained from it is more relevant for studying colloidal interactions [7].

We should also point out that, in a recently published work, fairly good agreement between the above mentioned prediction of Beresford-Smith's approach (non-monotonic behaviour with the surface charge) and experimental results was found for a set of liposome vesicles with different numbers of ionizable groups [8]. However, only moderately structured samples were observed for such systems and the probable failure of the theoretical model in the case of highly structured dispersions was claimed.

The aim of this work is to look into the non-monotonic behaviour of the spatial ordering with the surface charge in a set of model colloids and find out to what extent Beresford-Smith's approach can explain the observed structure factors in highly ordered systems. In order to characterize the evolution of static properties with the particle charge, we have studied several colloidal dispersions with similar particle size but different charges. The remainder of the work is organized as follows. First, the experimental systems and methods are described in some detail. Then, the experimental results are presented and compared to the theoretical predictions.

In our experiments, four latexes supplied by Ikerlat Polymers were used. Their sizes and numbers of ionizable sulfate groups (determined by means of transmission electron microscopy and titration, respectively) are shown in table 1. As can be seen, all these latexes have practically the same diameter. Their spherical form and monodispersity were also confirmed by fitting the corresponding form factors with the expression provided by the Rayleigh-GansDebye theory for spheres of identical size. 


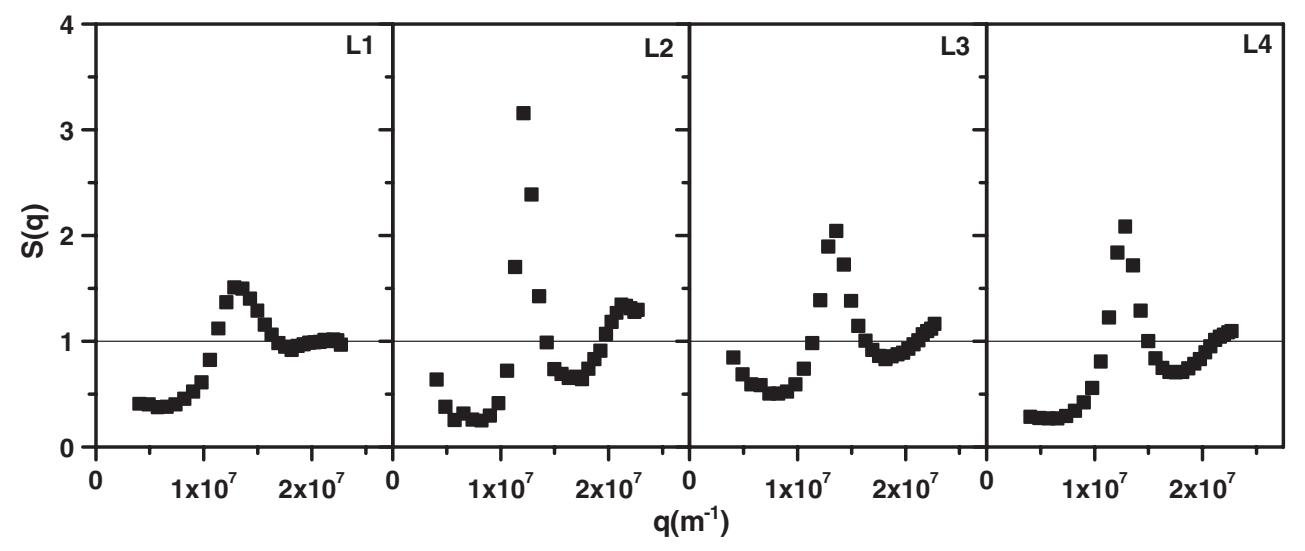

Figure 1. Experimental structure factors for latex systems at a volume fraction around $0.15 \%$.

The dependence on $q$ of the average scattered intensity was measured with a 3D-DLS system (LS Instruments) for angles from $15^{\circ}$ to $135^{\circ}$. The $3 \mathrm{D}$ cross-correlation scheme allows correcting the effect of multiple scattering on the scattered light intensity and can be used to successfully characterize turbid suspensions with transmission values, of the singly scattered light, as low as $0.01[9,10]$. The light source was a diode laser working at $680.4 \mathrm{~nm}$ and the device is provided with a multitau correlator (Flex). A complete description of the technique has been given elsewhere [9]. The different samples were prepared at $0.15 \%$ in volume fraction by dilution of stock solutions and kept for at least 20 days in a mixed bed of ion exchanger resin in sealed cylindrical quartz cuvettes. During this period, the scattered intensity was monitored and when no significant changes were observed, the deionization process was supposed to be completed. In figure 1, the structure factors observed for the four latexes are shown. First, the appearance of a re-entrant fluid-crystal-fluid phase upon increase of the number of charged groups per particle should be pointed out. Sample L2 shows crystallization. Nevertheless, in this work we focus on the metastable state of the samples. Due to the low particle number density of the system, of order $6 \times 10^{13} \mathrm{~cm}^{-3}$, the metastable state is obtained by tumbling the sample before each measurement [11]. Another striking feature of the measured $S(q)$ is the upturn at low values of $q$ for samples L2 and L3. In our case, the multiple scattering effects can be safely ruled out since the singly scattered light represents more than $90 \%$ of the measured intensity. Furthermore, even if the particle concentration in all the samples is very similar, the uprising only appears in these two samples. Rojas et al have observed a similar upturn in the static structure factor for latex samples at similar and higher concentrations, a feature that is also reflected in the dynamics [12].

Structure factors in figure 1 show that spatial correlation first increases with the number of ionizable groups $(N)$ and then seems to decrease. This non-monotonic behaviour can also be graphically illustrated by plotting the height of the first peak of $S(q), S_{\max }$, as a function of $N$ (see figure 2). $S_{\max }$ clearly exhibits a maximum versus $N$ (as predicted by BeresfordSmith et al; see figure 8 in [5]). However, it should be stressed that the value of $S_{\max }$ for the sample corresponding to this maximum (L2) is extremely large. In fact, this sample crystallizes after tumbling if we wait enough time, as mentioned above. According to the Verlet's rule, the crystallization takes place for $S_{\max }>2.8$ and this system is just over this threshold. In addition, we should mention that the crystallization of latex L2 was also observed (with the naked eye) for other particle concentrations. 


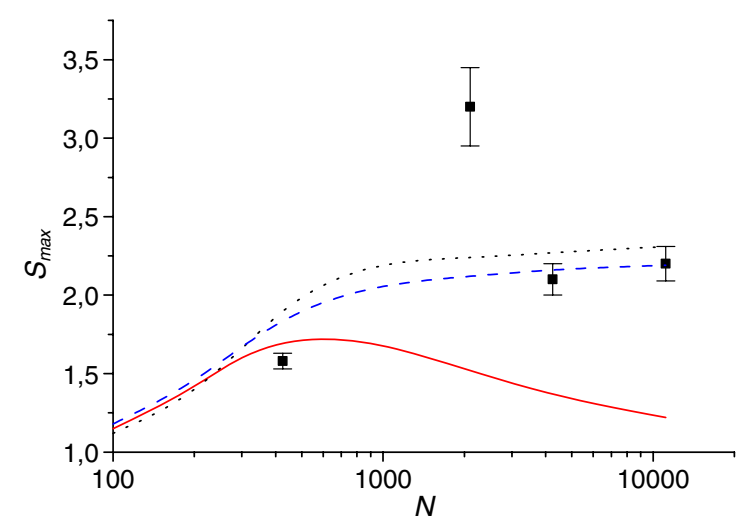

Figure 2. Height of the main peak of the structure factor, $S_{\max }$, as a function of the number of ionizable groups per particle. Squares denote the experimental results whereas solid, dotted and dashed lines indicate the predictions obtained from the jellium approximation, the cell model and the self-consistent modified jellium model (see equation (5)) respectively.

(This figure is in colour only in the electronic version)

As first attempt to explain our results we have applied the jellium renormalization scheme to our colloidal systems. In order to do that, the normalized electrostatic potential $\Psi$ must be calculated from the Poisson-Boltzmann (PB) equation assuming that: (i) colloidal particles are treated on the same footing as small ions; (ii) the spatial ordering is completely neglected, i.e. $g(r)=1$. Under these assumptions and for spherical particles, the PB equation then reads

$\Psi^{\prime \prime}(r)+\frac{2}{r} \Psi^{\prime}(r)=-4 \pi l_{\mathrm{B}}\left[\left(Z n_{\mathrm{P}}+n_{\mathrm{S}}\right) \exp (-\Psi)-n_{\mathrm{S}} \exp (\Psi)-Z n_{\mathrm{P}}\right] \quad(r \geqslant a)$

where $l_{\mathrm{B}}$ is the so-called Bjerrum length $\left(0.713 \mathrm{~nm}\right.$ for water at $\left.25^{\circ} \mathrm{C}\right)$, and $n_{\mathrm{P}}$ and $n_{\mathrm{S}}$ are the concentrations of colloidal particles and monovalent salt, respectively. Equation (2) is solved together with the boundary conditions $\Psi^{\prime}(a)=Z l_{\mathrm{B}} / a^{2}$, which is Gauss's law applied to the particle surface, and $\Psi(\infty)=0$. The far-field solution of equation (2) can be matched by the linearized solution

$$
\Psi_{0}(r)=l_{\mathrm{B}} \frac{Z_{\mathrm{ren}} \exp \left(\kappa_{\mathrm{ren}} a\right)}{1+\kappa_{\mathrm{ren}} a} \frac{\exp \left(-\kappa_{\mathrm{ren}} r\right)}{r}
$$

where $Z_{\text {ren }}$ and $\kappa_{\text {ren }}$ are the renormalized parameters. In the application of this procedure one could be tempted to identify the particle charge $(Z)$ with the total number of ionizable groups $(N)$. This assumption seems reasonable since we are dealing with strong acid groups (sulfate). However, in highly deionized suspensions the proton concentration close to the particle surface could be so extremely large that the local $\mathrm{pH}$ would even be comparable to the $\mathrm{p} K_{\text {sulfate }}$, as other authors pointed out [13]. Consequently, the actual number of dissociated surface groups $(Z)$ would be considerably smaller than the total one $(N)$, which can be estimated implementing a mass equation in our calculations. Accordingly, the mass equation for the dissociation of the surface sulfate groups would be $K_{\text {sulfate }}=\left[\mathrm{H}^{+}\right]_{0} \mathrm{Z} /(\mathrm{N}-\mathrm{Z})$, where $\left[\mathrm{H}^{+}\right]_{0}$ is the proton concentration at the particle surface. As this quantity depends on the surface electrostatic potential, this condition must be consistently applied together with the boundary condition derived from Gauss's law. Following some studies [13-15] $K_{\text {sulfate }} \approx 2$ was assumed. It should also be mentioned that, although our experiments are carried out with highly deionized samples, we have assumed a residual ion concentration of $10^{-6} \mathrm{M}$, following the estimations done by Evers et al [16]. In table 2, the results for $Z$ calculated from this procedure are 
Table 2. Values obtained for $Z$ (actual charge), $Z_{\text {ren }}$ (renormalized charge) and $\kappa_{\text {ren }} a$ from the jellium approximation and cell model.

\begin{tabular}{|c|c|c|c|c|c|c|}
\hline \multirow[b]{2}{*}{ Latex } & \multicolumn{2}{|c|}{$\begin{array}{c}Z \text { (in elementary } \\
\text { charges per particle) }\end{array}$} & \multicolumn{2}{|c|}{$\begin{array}{l}Z_{\text {ren }} \text { (in elementary } \\
\text { charges per particle) }\end{array}$} & \multicolumn{2}{|c|}{$\kappa_{\mathrm{ren}} a$} \\
\hline & Jellium & Cell model & Jellium & Cell model & Jellium & Cell model \\
\hline L1 & 407 & 411 & 285 & 352 & 0.224 & 0.240 \\
\hline L2 & 1153 & 1020 & 342 & 468 & 0.331 & 0.250 \\
\hline L3 & 1672 & 1326 & 345 & 482 & 0.389 & 0.251 \\
\hline $\mathrm{L} 4$ & 2780 & 1833 & 359 & 493 & 0.498 & 0.253 \\
\hline
\end{tabular}

shown. The values for $\kappa$ are also included. It should be noted that $\kappa$ is not renormalized in the jellium approach, in contrast to the cell model, and it depends on the bare charge $Z$, $\kappa=\sqrt{4 \pi l_{\mathrm{B}}\left(2 n_{\mathrm{S}}+Z n_{\mathrm{P}}\right)}$. This is the key point for understanding our results. After having calculated the parameters characterizing the interaction, $Z_{\text {ren }}$ and $\kappa_{\text {ren }}$, which are displayed in table 2, the structure factor and, more specifically, $S_{\max }$ can be computed. In our study, we applied the hypernetted chain (HNC) approximation where the input parameters are the renormalized charge $Z_{\text {ren }}$ (obtained from the dissociated charge, $Z$ ), $\kappa_{\text {ren }}$ and the volume fraction. In figure 2 the resulting $S_{\max }$ for a suspension of particles with $a=40 \mathrm{~nm}$ and $\varphi=0.0015$ is plotted to be compared with the experimental data. As can be seen, the prediction of the jellium approximation does not reproduce the experimental results. A nonmonotonic behaviour with $N$ is obtained but a quantitative description is not possible. On the one hand, it significantly underestimates the values of $S_{\max }$. Other authors have also reported such underestimation of the dependence of the spatial ordering on the volume fraction (or particle concentration) $[6,17]$. In other words, this theory overestimates the screening effect of the particles and/or underestimates the particle-particle spatial ordering. This is not surprising since the particle-particle structure has been completely neglected in this model, $g(r)=1$. On the other hand, certain disagreement between theory and experiment on the position of the maximum is also found.

At this point, we will also probe to what extent the classical Alexander cell model can capture the main features of the $S_{\max }-N$ plot. According to this model, $\Psi$ is computed solving the $\mathrm{PB}$ equation in a cell of radius $R$ (depending on the volume fraction):

$$
\Psi^{\prime \prime}(r)+\frac{2}{r} \Psi^{\prime}(r)=-4 \pi l_{\mathrm{B}}\left[\left(Z n_{\mathrm{P}}+n_{\mathrm{S}}\right) \exp (-\Psi)-n_{\mathrm{S}} \exp (\Psi)\right] \quad(a \leqslant r \leqslant R) .
$$

In this case, the presence of the other colloidal particles is considered, introducing the cell of finite volume. Again one assumes at $r=a$ that $\Psi^{\prime}(a)=Z l_{\mathrm{B}} / a^{2}$, whereas the electric field vanishes at the cell boundary (i.e., $\Psi^{\prime}(R)=0$ ), which guarantees electroneutrality. The renormalized parameters were calculated again approximating asymptotically the numerical solution of equation (4) at the WS cell boundary with a linearized solution of the PB equation. The dissociation of sulfate groups was taken into account as well. At any rate, it should be mentioned that Trizac et al have revisited the renormalization prescription of Alexander, suggesting an efficient way to apply such a scheme in practice [18]. Moreover, these authors have shed light on the definition and calculation of $\kappa$, which sometimes seems to be confused.

The results for the dissociated charge $Z$ and the renormalized charge $Z_{\text {ren }}$ obtained with the cell model are also shown in table 2. It should be emphasized that the Debye screening parameter is also renormalized, $\kappa_{\text {ren }}$, which did not occur in the case of the jellium approximation. Furthermore, $\kappa_{\text {ren }}$ seems to saturate with increasing $Z$. The predictions obtained for $S_{\max }$ (with $a=40 \mathrm{~nm}$ and $\varphi=0.0015$ ) are also plotted in figure 2. As expected, this 
quantity increases monotonically with increasing particle charge and, consequently, this model also fails to fully describe our data. From a quantitative viewpoint, these predictions seem to be somewhat successful, with the clear exception of the crystallizing sample ones.

Certainly, figure 2 suggests that the jellium approximation overestimates the screening of the macroions (whose actual charge is $Z$ ), so we intended to apply a modified jellium model improving this picture and inspired by an idea put forward by Trizac and Levin [7]. According to these authors, one should suppose that colloidal particles screen with their renormalized (or effective) charge instead of the actual one. Consequently, equation (2) could be rewritten as

$\Psi^{\prime \prime}(r)+\frac{2}{r} \Psi^{\prime}(r)=-4 \pi l_{\mathrm{B}}\left[\left(Z_{\mathrm{ren}} n_{\mathrm{P}}+n_{\mathrm{S}}\right) \exp (-\Psi)-n_{\mathrm{S}} \exp (\Psi)-Z_{\mathrm{ren}} n_{\mathrm{P}}\right] \quad(r \geqslant a)$

and $\kappa=\sqrt{4 \pi l_{\mathrm{B}}\left(2 n_{\mathrm{S}}+Z_{\mathrm{ren}} n_{\mathrm{P}}\right)}$. Obviously, the problem then is that $Z_{\text {ren }}$ is unknown $a$ priori. In fact, we must solve equation (5) (and then apply equation (3)) to determine the renormalized charge. In other words, this parameter is calculated self-consistently (together with the corresponding boundary conditions and the mass equation for the dissociation of the surface sulfate groups). First, a trial value for $Z_{\text {ren }}$ is chosen (as input). After solving the previous differential equation, this guess is compared with the output value. This procedure is repeated until both coincide. The results are also plotted in figure 2. As can be seen, the behaviour is quite similar to that reported for the cell model, although the quantitative agreement with experimental data is better. In any case, this self-consistent jellium model cannot explain the crystallization of sample L2 either.

Other authors have also investigated the influence of the particle charge on the solid-liquid phase transition for silica and polymer latex dispersions [19], although for a narrow range of surface charge densities $\left(<1 \mu \mathrm{C} \mathrm{cm}^{-2}\right)$. They also reported a re-entrant transition with increasing surface charge density and claimed that such behaviour is not explicable in terms of a purely repulsive Yukawa potential complemented with a charge renormalization scheme. This study seems to support this conclusion from an analysis in which we have explicitly considered: (i) the measure of the structure factor for characterizing the spatial ordering; (ii) two models for predicting renormalized interaction parameters. The theory proposed by Beresford-Smith et al is able to predict the weakening of the spatial correlation with increasing particle charge but the jellium approximation is inadequate for highly structured samples. As regards the cell model, this scheme cannot predict the crystallization of one of the samples. In our opinion, the existence of an attractive contribution to the interaction potential is still a controversial issue. For instance, Roij et al have shown that the observation of a fluid-fluid phase separation in a charge-stabilized colloidal dispersion does not necessarily imply such attractive component in the effective pair interaction between highly charged particles [20]. On the other hand, some of the same authors have considered three-body forces finding attractive triplet interactions [21]. Nevertheless, the structure factors reported here show a feature that could be attributed to an attractive component, i.e. the increase in $S(q)$ for low $q$ (see figure 2). In a previous work, polydispersity and the presence of large aggregates were also considered causes for such increase [22]. However, the latexes used here are quite monodisperse, less than $5 \%$. Concerning the existence of aggregation, it should be noted that the increase in $S(q)$ for low $q$ is also present (and even more noticeable) for the crystallizing sample (L2). As the crystallization in this sample is the result of very intense electrostatic forces, aggregation due to electrostatic destabilization does not seem feasible. Consequently, this phenomenon should also be rejected as the cause for the behaviour of the structure factor at low $q$.

In our opinion, the rise of the intensity observed for small scattering vectors may be related to the proximity of the phase transition mentioned above (fluid-crystal). The presence of small 
domains of crystals in the micron size range may result in an additional low $q$ contribution to the scattered intensity. Whichever is the case, our finding confirms the existence of spatial heterogeneities. Extensive studies in the low $q$ regime should be carried out in order to rule out this scenario.

We have observed a non-monotonic dependence of the local order on the particle charge, which has been analysed in terms of two renormalization schemes. The jellium approximation explains qualitatively the existence of a certain charge where the correlation among particles is maximum but it underestimates the height of the structure factor main peak. In contrast, the cell model, which seems to improve the prediction of the experimental $S_{\max }$ values at larger particle charges, cannot explain the non-monotonic behaviour.

We thank the 'Ministerio de Ciencia y Tecnología, Plan Nacional de Investigación, Desarrollo e Innovación Tecnológica $(\mathrm{I}+\mathrm{D}+\mathrm{I})$ ' for financial support. In particular, CHP, JCF, MQP and RHA are indebted to project MAT2003-08356-CO4-01 and to the European Regional Development Fund (ERDF). MQP is also grateful for financial support from 'Consejería de Innovación, Ciencia y Tecnología de la Junta de Andalucía'. Finally, CHP also acknowledges useful discussions with Veronique Trappe and Luis Rojas.

\section{References}

[1] Belloni L 2000 J. Phys.: Condens. Matter 12 R549

[2] Hansen J P and Löwen H 2000 Annu. Rev. Phys. Chem. 51209

[3] Quesada-Pérez M, Callejas-Fernández J and Hidalgo-Álvarez R 2002 Adv. Colloid Interface Sci. 95295

[4] Alexander S, Grant P M, Morales G J, Pincus P and Horne D 1984 J. Chem. Phys. 805776

[5] Beresford-Smith B, Chan D Y C and Michel D J 1985 J. Colloid Interface Sci. 105216

[6] Rojas L F, Urban C, Schurtenberger P, Gisler T and Von Grünberg H H 2002 Europhys. Lett. 60802

[7] Trizac E and Levin Y 2004 Phys. Rev. E 69031403

[8] Haro-Pérez C, Quesada-Pérez M, Callejas-Fernández J, Sabaté E, Estelrich J and Hidalgo-Álvarez R 2005 Colloids Surf. A 270352

[9] Urban C and Schurtenberger P 1998 J. Colloid Interface Sci. 207150

[10] Overbeck E, Sinn C and Palberg T 1997 Prog. Colloid Polym. Sci. 104117

[11] Van Megen W and Pusey P N 1991 Phys. Rev. A 435429

[12] Rojas L F 2004 PhD Thesis Institut de Physique, Université de Fribourg

[13] Palberg T, Kottal J, Bitzer F, Simon R, Würth M and Leiderer P 1995 J. Colloid Interface Sci. 16985

[14] Zwetsloot J P H and Leyte J C 1994 J. Colloid Interface Sci. 163362

[15] Jacobasch H J 1984 Die Angew. Makrom. Chem. 12847

[16] Evers M, Garbow N, Hessinger D and Palberg T 1998 Phys. Rev. E 576774

[17] Härtl W and Versmold H 1988 J. Chem. Phys. 887157

[18] Trizac E, Bocquet L, Aubouy M and von Grünberg H H 2003 Langmuir 194027

[19] Yamaka J, Yoshida H, Koga T, Ise N and Hashimoto T 1998 Phys. Rev. Lett. 805806

[20] Van Roij R, Dijkstra M and Hansen J P 1999 Phys. Rev. E 592010

[21] Russ C, Von Grünberg H H, Dijkstra M and Van Roij R 2002 Phys. Rev. E 66011402

[22] Haro-Pérez C, Quesada-Pérez M, Callejas-Fernández J, Casals E, Estelrich J and Hidalgo-Álvarez R 2003 J. Chem. Phys. 1185167 